\title{
L-Amino Acid Oxidases with Specificity for Basic L-Amino Acids in Cyanobacteria
}

\author{
Achim E. Gau ${ }^{\mathrm{a}}$, Achim Heindl ${ }^{\mathrm{b}}$, Anke Nodop ${ }^{\mathrm{b}}$, Uwe Kahmann ${ }^{\mathrm{b}}$, \\ and Elfriede K. Pistorius ${ }^{\mathrm{b}, *}$ \\ a Institut für Botanik, Gottfried Wilhelm Leibniz Universität Hannover, \\ D-30419 Hannover, Germany \\ b Biologie VIII: Molekulare Zellphysiologie, Universität Bielefeld, D-33501 Bielefeld, \\ Germany. E-Mail: e.pistorius@uni-bielefeld.de \\ * Author for correspondence and reprint requests \\ Z. Naturforsch. 62 c, 273-284 (2007); received October 23/November 24, 2006
}

The two closely related fresh water cyanobacteria Synechococcus elongatus PCC 6301 and Synechococcus elongatus PCC 7942 have previously been shown to constitutively express a FAD-containing L-amino acid oxidase with high specificity for basic L-amino acids (L-arginine being the best substrate). In this paper we show that such an enzyme is also present in the fresh water cyanobacterium Synechococcus cedrorum PCC 6908. In addition, an improved evaluation of the nucleotide/amino acid sequence of the L-amino acid oxidase of Synechococcus elongatus PCC 6301 (encoded by the aoxA gene) with respect to the FAD-binding site and a translocation pathway signal sequence will be given. Moreover, the genome sequences of 24 cyanobacteria will be evaluated for the occurrence of an aoxA-similar gene. In the evaluated cyanobacteria 15 genes encoding an L-amino acid oxidase-similar protein will be found.

Key words: Cyanobacteria, L-Amino Acid Oxidase, Synechococcus elongatus PCC 6301 and PCC 7942

\section{Introduction}

The two closely related cyanobacteria Synechococcus elongatus PCC 6301 and Synechococcus elongatus PCC 7942 have previously been shown to possess a FAD-containing L-amino acid oxidase (L-Aox) catalyzing the oxidative deamination of basic L-amino acids (L-Arg $>$ L-Lys $>$ L-Orn $>$ LHis) by utilizing $\mathrm{O}_{2}$ as electron acceptor resulting in the formation of the corresponding ketoacid, ammonium, and hydrogen peroxide (Pistorius et al., 1979; Pistorius and Voss, 1980; Engels et al., 1992). Another rather unusual property, besides having a high specificity for basic L-amino acids, is its strong inhibition by cations $\left(\mathrm{M}^{3+}>\mathrm{M}^{2+}>\mathrm{M}^{+}\right)$ and less strong inhibition by anions. In the group of divalent cations, transition metal ions (like $\mathrm{Mn}^{2+}$ ) inhibit more strongly than alkaline-earth metal ions (like $\mathrm{Ca}^{2+}$ ). The L-Aox in Synechococcus elongatus PCC 6301 and PCC 7942 is in part located in the soluble protein fraction of the periplasm and in part in the spheroplast fraction, mainly precipitating with the membrane fraction (Bockholt et al., 1996 and unpublished results).

Abbreviations: L-Aox, L-amino acid oxidase; aox $A, \mathrm{~L}-$ amino acid oxidase encoding gene.
The L-Aox encoding gene, called aox $A$, has previously been identified in Synechococcus elongatus PCC 6301. A preliminary nucleotide sequence was published by Bockholt et al. (1995) and a corrected nucleotide sequence and the derived amino acid sequence were later submitted to the data base under the accession number Z48565 with an entry date of July 1998.

Construction of an L-Aox-free Synechococcus elongatus PCC 7942 mutant and subsequent partial characterization of this mutant revealed that in contrast to the wild-type the mutant could not grow on L-arginine as sole nitrogen source suggesting that this L-Aox is the only enzyme that enables the cells to utilize extracellularly added L-arginine as N-source (Bockholt et al., 1996). However, since Synechococcus elongatus PCC 7942 can not take up L-arginine effectively (Montesinos et al., 1997), this result does not exclude that other L-arginine-metabolizing enzymes are present intracellularly.

Besides being a constituent of proteins, L-arginine has an additional role in some cyanobacteria, which have an alternative $\mathrm{CO}_{2}$ fixation pathway via carbamoyl phosphate leading to citrulline and subsequently to arginine (Linko et al., 1957; Ta- 
bita, 1994) in addition to the major $\mathrm{CO}_{2}$ fixation reaction via Rubisco in the Calvin cycle. Moreover, it is well documented that a number of cyanobacteria are able to synthesize the polymer cyanophycin, which consists of an aspartic acid backbone with L-arginine residues attached to the $\beta$-carboxy group of aspartate by isopeptide bonds (Simon, 1971, 1987; Allen, 1984). The peptide as well as the isopeptide bonds are synthesized by the cyanophycin synthetase encoded by the $c p h A$ gene (Ziegler et al., 1998; Hai et al., 1999; Berg et al., 2000; Aboulmagd et al., 2000). In the early literature it has been stated that the two closely related Synechococcus elongatus PCC 6301 and PCC 7942 are not able to synthesize cyanophycin (Simon, 1987), and later it has been shown that these two cyanobacteria do not possess the cphA gene (Ziegler et al., 1998). Thus, they are unable to store L-arginine in the cyanophycin polymer. However, they constitutively express the above described LAox, which converts L-arginine to 2-ketoarginine being further metabolized to guanidinobutyrate by a nonenzymatic reaction when hydrogen peroxide is not removed by hydrogen peroxide decomposing enzymes.
In this paper, we report on an additional cyanobacterium, besides Synechococcus elongatus PCC 6301 and PCC 7942, which has such an L-Aox being readily detectable by a stimulation of the $\mathrm{O}_{2}$ uptake by intact cells when L-arginine is added as substrate. In addition an extended characterization of the L-Aox sequence of Synechococcus elongatus PCC 6301 with respect to the FAD-binding site and a translocation pathway signal sequence are performed. Moreover, on the basis of the LAox sequence of Synechococcus elongatus PCC 6301 (encoded by the aox $A$ gene) the genome sequences of 24 cyanobacteria are evaluated for the presence of a gene encoding such a putative L-Aox with specificity for basic L-amino acids.

\section{Materials and Methods}

Origin of cyanobacteria, growth, French press extract preparation, L-Aox activity determination, and immunocytochemical investigation

Synechococcus elongatus PCC 6301 was obtained from the Sammlung von Algenkulturen der Universität Göttingen, Göttingen, Germany, and Synechococcus elongatus PCC 7942 as well as Syn-

Table I. The origin of the evaluated cyanobacterial genome sequences.

\begin{tabular}{ll}
\hline Cyanobacterial strain & Origin of genome sequence $^{\mathrm{a}}$ \\
\hline Prochlorococcus marinus SS 120 & European Union / Genoscope \\
Prochlorococcus marinus MIT 9211 & Craig Venter Institute \\
Prochlorococcus marinus MIT 9312 & JGI / MIT / DOE \\
Prochlorococcus marinus MIT 9313 & JGI / DOE \\
Prochlorococcus marinus MED 4 & JGI / DOE \\
Prochlorococcus marinus NATL 2A & JGI / MIT / DOE \\
Synechococcus sp. WH 8102 & JGI / DOE \\
Synechococcus sp. CC 9902 & JGI / DOE \\
Synechococcus sp. RS 9917 & Craig Venter Institute \\
Synechococcus sp. CC 9605 & JGI / DOE \\
Synechococcus sp. WH 5701 & Craig Venter Institute \\
Synechococcus sp. WH 7805 & Craig Venter Institute \\
Trichodesmium erythraeum IMS 101 & WHOI / JGI / DOE \\
Crocosphaera watsonii WH 8501 & WHOI / JGI/ DOE \\
Synechococcus elongatus PCC 6301 & Nagoya University \\
Synechococcus elongatus PCC 7942 & JGI / Texas A \& M University / DOE \\
Synechocystis sp. PCC 6803 & Kazusa DNA Research Institute \\
Gloeobacter violaceus PCC 7421 & Kazusa DNA Research Institute \\
Nostoc sp. PCC 7120 & Kazusa DNA Research Institute \\
Nostoc punctiforme PCC 73102 & JGI / DOE \\
Anabaena variabilis ATCC 29413 & Missouri State University / JGI / DOE \\
Thermosynechococcus elongatus BP-1 & Kazusa DNA Research Institute \\
Synechococcus Yellowstone A (JA-3-3Ab) & TIGR \\
Synechococcus Yellowstone B (JA-2-3B'a(2-13)) & TIGR \\
\hline
\end{tabular}

a JGI, Joint Genome Research Institute; DOE, Department of Energy USA; MIT, Massachusetts Institute of Technology; WHOI, Woods Hole Oceanographic Institute; TIGR, The Institute for Genomic Research. 
echococcus cedrorum PCC 6908 were obtained from the Pasteur Culture Collection of Cyanobacterial Strains, Paris, France. Growth and French press extract preparation were as described in Yousef et al. (2003). The L-Aox activity was determined as described in Engels et al. (1992), and the immunocytochemical investigation was done as described in Bockholt et al. (1996) utilizing the antiserum raised against the isolated L-Aox from Synechococcus elongatus PCC 6301.

\section{DNA isolation, cloning, and nucleotide sequencing}

For a re-evaluation of the aox $A$ sequence, the previously obtained $10 \mathrm{~kb}$ EcoRI/Bam HI chromosomal DNA fragment (pDL100) from Synechococcus elongatus PCC 6301, which carries the aoxA gene (Bockholt et al., 1995), was digested into a $1.9 \mathrm{~kb}$ PstI fragment (pDV18) and a $1.5 \mathrm{BglII}$ fragment (pDL14). For sequencing of these two overlapping strands the fragments were cloned into pUC19 and pSVB30, respectively.

\section{Genome sequence evaluation and analyses}

Nucleic acid and the derived protein sequences were analyzed using the Staden software package (Staden, 1986). The deduced amino acid sequences were analyzed using two different programs: PC/ Gene (IntelliGenetics Release 6.8, Camino Real Mountain View, California, 1993) and XPIP (Staden, 1986). Homology searches were done with the Blast algorithm by Altschul et al. (1990) in Swiss-PROT, EMBL, PIR, GenBank, and CyanoBase databases. Sequence alignments were performed with ClustalW (Thompson et al., 1994), and calculations of identities and similarities were performed according to Tatusova and Madden (1999). Prediction of putative signal sequences were done with SMART (Ponting et al., 1999; Schultz et al., 1998). The origin of the cyanobacterial genome sequences are listed in Table I.

\section{Results and Discussion}

Presence of an L-Aox with specificity for basic L-amino acids in Synechococcus cedrorum PCC 6908

In the early literature it has been stated that besides the two mesophilic cyanobacteria, Synechococcus elongatus PCC 6301 and Synechococcus elongatus PCC 7942, the mesophilic cyanobacterium Synechococcus cedrorum PCC 6908 also is not able to synthesize cyanophycin (Simon, 1987). Since all three cyanobacteria are fresh water cyanobacteria growing only under photoautotrophic conditions, we investigated whether Synechococcus cedrorum PCC 6908 also contains an L-Aox with high specificity for basic L-amino acids. As the results of Table II show, this is indeed the case. The activity of this enzyme was readily detectable in cell suspensions or cell extracts by a stimulation of the $\mathrm{O}_{2}$ uptake when L-arginine was added. The properties of the L-Aox in Synechococcus cedro-

Table II. Comparison of the L-amino acid oxidase in Synechococcus elongatus PCC 6301, Synechococcus elongatus PCC 7942, and Synechococcus cedrorum PCC 6908. The L-Aox activity was determined in French press extracts (FPE) prepared from a cell suspension containing $100 \mu \mathrm{l}$ cells $/ \mathrm{ml}$. Growth of cells was for two days in BG11 medium. Activity measurements were performed as described in Materials and Methods.

\begin{tabular}{|c|c|c|c|}
\hline & $\begin{array}{l}\text { S. elongatus } \\
\text { PCC } 6301\end{array}$ & $\begin{array}{l}\text { S. elongatus } \\
\text { PCC } 7942\end{array}$ & $\begin{array}{l}\text { S. cedrorum } \\
\text { PCC } 6908\end{array}$ \\
\hline $\begin{array}{l}\text { L-Aox activity in French press extracts } \\
{\left[\mu \mathrm{mol} \mathrm{O}_{2} \text { taken up/ml FPE } \times \mathrm{h}\right]} \\
(\mathrm{L} \text {-arginine as substrate })\end{array}$ & $60-80$ & $10-15$ & $45-70$ \\
\hline$K_{\mathrm{M}}$ value for L-arginine $[\mathrm{mm}]$ & 5 & 3 & 4 \\
\hline $\mathrm{CaCl}_{2}$ inhibition: $I_{50}[\mathrm{~mm}]$ & 1.6 & 1.1 & 1.1 \\
\hline $\begin{array}{l}\text { Relative substrate specifity }(\%) \\
\text { L-Arginine }=100 \% \text { activity } \\
\text { L-Lysine } \\
\text { L-Ornithine } \\
\text { L-Histidine }\end{array}$ & $\begin{array}{r}100 \\
73 \\
46 \\
<2\end{array}$ & $\begin{array}{r}100 \\
82 \\
28 \\
<2\end{array}$ & $\begin{array}{r}100 \\
81 \\
35 \\
<2\end{array}$ \\
\hline
\end{tabular}

The L-Aox, which is characterized in this table, is encoded in Synechococcus elongatus PCC 6301 and PCC 7942 by the aoxA gene (see Table IV). The gene sequence of the L-Aox of Synechococcus cedrorum PCC 6908 is not yet available. 
rum PCC 6908 are very similar to the properties of the L-Aox in Synechococcus elongatus PCC 6301 and PCC 7942 with respect to substrate specificity, $K_{\mathrm{M}}$ value for L-arginine, and inhibition by cations (shown for $\mathrm{CaCl}_{2}$ inhibition). Moreover, in all three Synechococcus species the L-Aox is expressed constitutively when cells are grown with nitrate as $\mathrm{N}$-source. The amount of the L-Aox activity detectable in cell extracts was somewhat variable (a range is given in Table II), but in all tested preparations the highest activity was observed in Synechococcus elongatus PCC 6301 and the lowest activity in Synechococcus elongatus PCC 7942. As in Synechococcus elongatus PCC
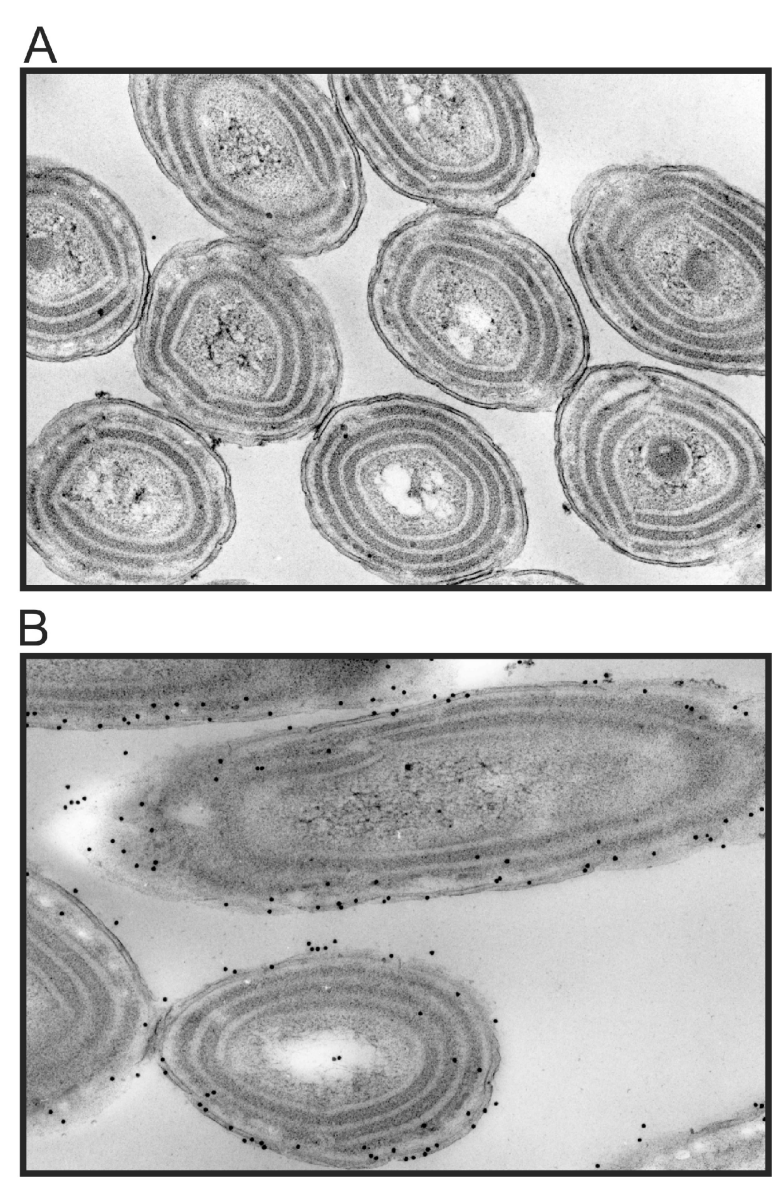

Fig. 1. Immunocytochemical detection of the L-amino acid oxidase in Synechococcus elongatus PCC 7942. Immunocytochemical detection of the L-Aox (encoded by the aox A) with the anti-L-Aox antiserum (Engels et al., 1992) in combination with gold-labeled anti-rabbit IgG. The cells were grown in BG11 medium for 3 days. A, Control serum; B, anti-L-Aox antiserum.
6301 and PCC 7942, the L-Aox in Synechococcus cedrorum PCC 6908 is in part located in the soluble protein fraction of the periplasm and in part in the spheroplast fraction. On activity basis about 40 to $60 \%$ of the total activity was detected in the soluble protein fraction of the periplasm (not shown).

Immunocytochemical investigations have confirmed the partially extracellular localization of the L-Aox in the investigated mesophilic Synechococcus species as previously shown for Synechococcus elongatus PCC 6301 (Bockholt et al., 1996) and as shown in Fig. 1 for the L-Aox of Synechococcus elongatus PCC 7942.

Nucleotide/amino acid sequence analysis of the aoxA gene of Synechococcus elongatus PCC 6301 encoding an L-Aox with specificity for basic L-amino acids

Previously a $10 \mathrm{~kb}$ EcoRI/BamHI chromosomal DNA fragment (pDL100) from Synechococcus elongatus PCC 6301 was shown to carry the L-Aox encoding gene, which was called aoxA (Bockholt et al., 1995). The sequence of part of this DNA fragment, after digestion with $B g l \mathrm{II}$ or PstI resulting in two overlapping DNA fragments named pDL14 and pDV18, was re-evaluated. The re-sequenced DNA region (consisting of 2993 bp with a GC content of $53.5 \%$ - data base accession number Z48565 - entry date July 1998) contains the complete aoxA gene (orf2: bp 1062 to 2549) and two incomplete ORFs transcribed in opposite direction to the aoxA: orf1 (bp 727 to 1) located upstream of the aoxA and orf3 (bp 2993 to 2568) located downstream of the aoxA. The aoxA sequence given under the accession number Z48565 is $100 \%$ identical with the sequence of gene YP_171306 of the whole genome sequence of Synechococcus elongatus PCC 6301 (Sugita: Nagoya University - CanoBase entry in December 2004) and also $100 \%$ identical with the sequence of gene ZP_00164087 of the recently available whole genome sequence of Synechococcus elongatus PCC 7942 (JGI/Texas A \& M University/DOE - 2005). Synechococcus elongatus PCC 6301 and Synechococcus elongatus PCC 7942 are two cyanobacteria, which are very closely related (Golden et al., 1989).

Evaluation of the re-sequenced nucleotide sequence of the aox $A$-carrying DNA region led to the conclusion that the aoxA gene consists of 1488 
nucleotides including the stop codon TGA at position bp 2547 to 2549 (GC content of $57.5 \%$ ). Of the three possible start codons: ATG starting at bp 1062, TTG starting at bp 1134, and GTG starting at bp 1188, GTG can be excluded, since it is located in the FAD-binding site (see below). Since ATG is the most frequently used start codon in cyanobacteria and since 11 bp upstream of this ATG a putative Shine-Dalgarno core sequence (1047-AGGA-1050) is located, the most likely start of the $\operatorname{aoxA}$ is at bp 1062 . So far no typical promoter region(s) could be identified. Based on this assumption the derived L-Aox protein consists of 495 amino acids with a calculated molecular mass of $54131 \mathrm{Da}$ (the native enzyme is a homodimer) and an isoelectric point of 8.52 (PC Gene). 17 amino acids have been verified by partially sequencing of the isolated L-Aox protein from Synechococcus elongatus PCC 6301 (amino acid residues 101 to 117) (Bockholt et al., 1995).

Characteristics of the FAD-binding motif

Previously, we have shown that the L-Aox from Synechococcus elongatus PCC 6301 as well as from PCC 7942 contain noncovalently bound FAD as a prosthetic group (Pistorius and Voss, 1980; Engels et al., 1992). The peptide fragment between amino acid residues 42 to 70 in Synechococcus elongatus PCC 6301 contains the highly conserved motif of a dinucleotide binding site according to Wierenga et al. (1986) (Fig. 2). This binding site (consisting of 29 to 31 residues in a number of investigated FAD-containing enzymes) can be recognized by a common fingerprint which consists of a set of 11 rules describing the type of amino acid that should occur in a specific position. While the three glycine residues as well as the acid residue at the end of the fingerprint are strictly conserved, the others can be changed according to the rules (Wierenga et al., 1986; Eggink et al., 1990). The L-Aox fragment consists of 29 residues (amino acids 42 to $70)$ and contains the three conserved glycines (GxGxxG at position 47 to 52), the conserved glutamic acid residue at the end of the motif as well as the other seven residues of the fingerprint resulting in the maximum fingerprint score of 11 . Secondary structure analysis for the L-Aox predicts the expected $\beta \alpha \beta$-fold with the loop positioned between the $\alpha$-helix and the second $\beta$-sheet (Fig. 2). Comparison of the Synechococcus L-Aox sequence with the sequence of FAD-containing monoamine oxidases, which have recently been analyzed with respect to additional functional regions for FAD binding (see review: Abell and Kwan, 2001), led to the identification of the fingerprint site being most likely involved in binding of the ribityl chain of FAD and of a $C$-terminal region being part of the active site (marked by $\mathrm{A}$ and B in Fig. 2, respectively).

One additional aspect should be mentioned. We observed substantial modifications of FAD leading to modified absorbance spectra of FAD in some of the purified enzyme fractions and as a consequence to substantial inactivation of the enzyme (Wälzlein et al., 1988). Since a methionine residue is present in the FAD-binding site (see Fig. 2) and since methionine can readily become oxidized by hydrogen peroxide (being a product of the L-Aox reaction) to methionine sulfoxide and further to methionine sulfone (Kachurin et al., 1995), it is likely that in some enzyme molecules FAD became modified by interaction with the oxidized methionine residue in the active centre of the enzyme.

Prediction of a possible translocation pathway signal

Bacterial proteins, which contain a redox factor of seven different types including FAD and which are transported into the periplasm, frequently have an unusual long signal sequence bearing the consensus S/T-R-R-x-F-L-K motif immediately before the hydrophobic region. According to Berks (1996) the twin-arginines are completely invariant, and the frequency of occurrence of each of the other amino acids exceeds $50 \%$. The L-Aox of Synechococcus elongatus PCC 6301 has such a twin-arginine translocation pathway signal sequence of 32 amino acid residues with a predicted cleavage site behind the glutamine in position 32 (according to program SMART). The corresponding motif in Synechococcus elongatus PCC 6301 is -S-R-R-R-F-L-Q- (amino acid residues 4 to 10, see Fig. 2) with only one amino acid not following the rule ( $\mathrm{Q}$ instead of $\mathrm{K})$. Cleavage of the L-Aox behind glutamine 32 would result in a mature protein of $50896 \mathrm{Da}$ having an isoelectric point of 6.2. Thus, the cleaved protein is an acidic protein in contrast to the uncleaved protein with a $\mathrm{pI}$ of 8.5. The presence of two forms of the L-Aox protein in the cell is supported by the observation that the uncleaved protein binds tightly to CM Sepharose at $\mathrm{pH} 5.5$, while the cleaved form does not. Both forms catalyze the oxidative deamination of basic 


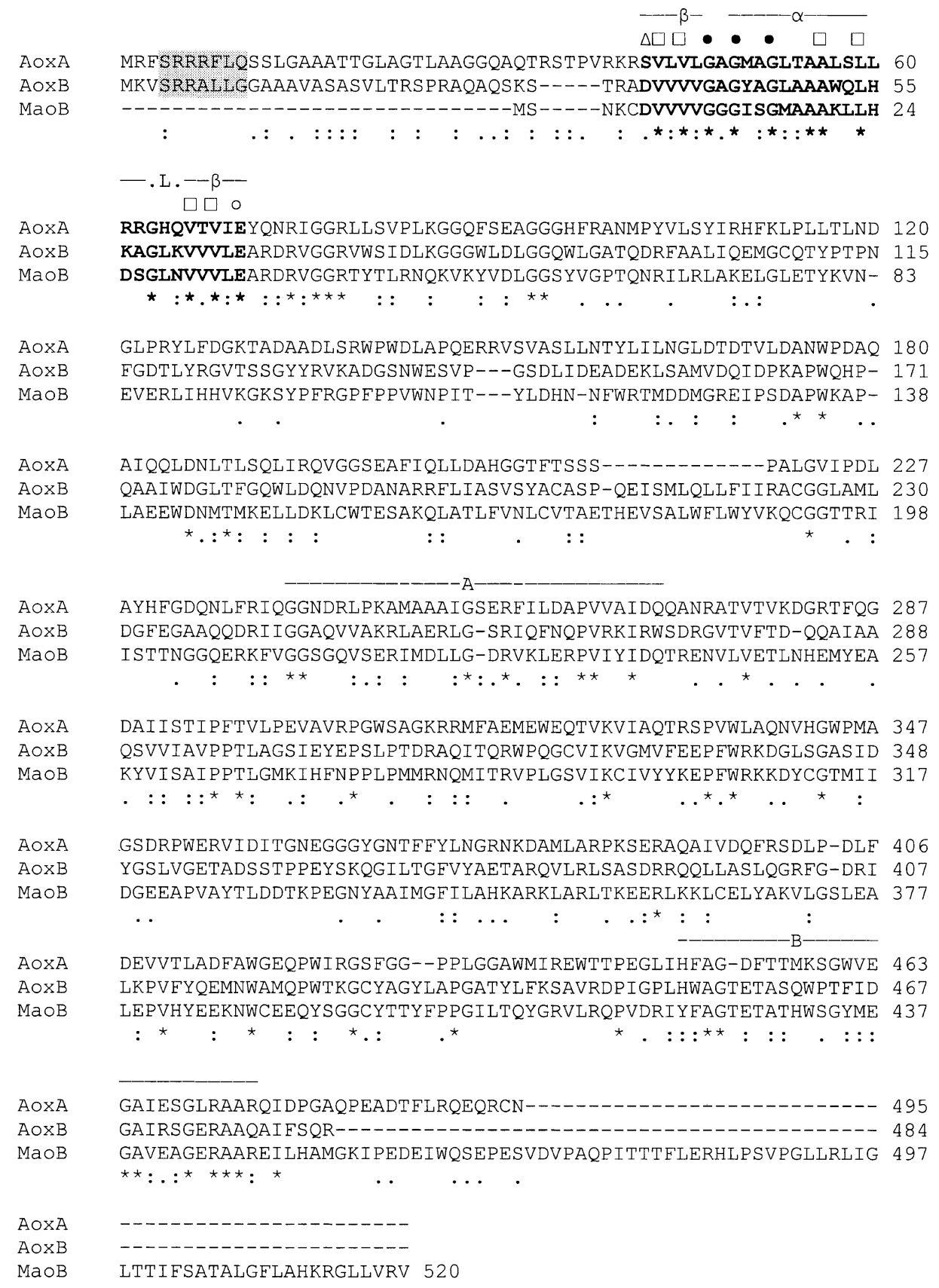

Fig. 2. Alignment of the AoxA (accession number YP_171306) and the L-AoxB (accession number YP_171854) of Synechococcus elongatus PCC 6301 with the monoamine oxidase B from human (MAO B - Swiss Prot accession number P27338). The asterisks mark identical residues, double points mark conserved substitutions, and single points mark semiconserved substitutions. The fingerprint of the dinucleotide-binding site of FAD according to Wierenga et al. (1986) is shown together with the prediction of the two $\beta$-sheets and the $\alpha$-helix. Bold letters, dinucleotidebinding site; $\Delta$, basic or hydrophilic amino acid residue; $\square$, small and hydrophobic amino acid residue; $\bullet$, highly conserved glycine residue; O, highly conserved acidic amino acid residue; $-\beta$-, $\beta$-sheets; - $\alpha$-, $\alpha$-helix; L, loop; -A-, additional amino acid region contributing to FAD-binding; -B-, part of the presumed active site. Moreover, a twinarginine translocation pathway signal is marked with grey colour. 
L-amino acids with $\mathrm{O}_{2}$ as electron acceptor (not shown).

It is also well documented in the literature (see e.g. Wexler et al., 1998; Brink et al., 1998) that a twin-arginine motif immediately prior to a hydrophobic region is also an essential feature for translocation of proteins across the thylakoid membrane in a Sec-independent transport system in plant chloroplasts. Another protein that we investigate in our group is a protein called IdiA whose expression greatly increases under iron limitation in Synechococcus elongatus PCC 6301 and PCC 7942 (Michel and Pistorius, 2004). This protein also carries a putative twin-arginine translocation pathway signal in the $\mathrm{N}$-terminal region being -SR-R-D-F-L-L- with also only one amino acid not following the rule ( $\mathrm{L}$ instead of $\mathrm{K}$ ). This protein is intracellularly located and is mainly attached to the thylakoid membrane. As suggested in the literature (Wan et al., 1996; Kouranov and Schnell, 1996), this implies that the passenger protein behind such a signal peptide also has an influence on the localisation of the protein.
Since the L-Aox in Synechococcus elongatus PCC 6301 (and also in Synechococcus elongatus PCC 7942) has been shown to be in part located in the soluble protein fraction of the periplasm, the presence of a putative translocation pathway signal is in agreement with this location. However, a substantial part of the enzyme remains intracellular. This finding can possibly be best explained by a partial translocation of the L-Aox into the periplasm (via the cytoplasmic membrane) and a partial transport of the L-Aox to the thylakoid membrane being there most likely loose associated with the cytoplasmic side of this membrane.

\section{Evaluating of 24 cyanobacterial genome sequences for the presence of an aoxA-similar gene}

We evaluated the genomes of 24 cyanobacteria, which are listed in Table III, for the presence of a gene encoding a protein with similarity to the L-Aox (encoded by the aox $A$ gene) with specificity
Cyanobacterium

Presence $(+)$ or absence $(-)$ of a gene with similarity to

$\operatorname{aox} A \quad \operatorname{cph} A$

Marine cyanobacteria:

Prochlorococcus marinus SS 120

Prochlorococcus marinus MIT 9211

Prochlorococcus marinus MIT 9312

Prochlorococcus marinus MIT 9313

Prochlorococcus marinus MED 4

Prochlorococcus marinus NATL 2A

Synechococcus sp. WH 8102

Synechococcus sp. CC 9902

Synechococcus sp. RS 9917

Synechococcus sp. CC 9605

Synechococcus sp. WH 5701

Synechococcus sp. WH 7805

Trichodesmium erythraeum IMS 101

Crocosphaera watsonii WH 8501

\begin{tabular}{|c|c|}
\hline - & - \\
\hline- & - \\
\hline- & - \\
\hline- & - \\
\hline- & - \\
\hline- & - \\
\hline- & - \\
\hline- & - \\
\hline- & - \\
\hline++ & - \\
\hline+ & - \\
\hline+ & - \\
\hline+ & + \\
\hline- & + \\
\hline
\end{tabular}

Fresh water cyanobacteria:

Synechococcus elongatus PCC 6301

Synechococcus elongatus PCC 7942

Synechocystis sp. PCC 6803

Gloeobacter violaceus PCC 7421

Nostoc sp. PCC 7120

Nostoc punctiforme PCC 73102

Anabaena variabilis ATCC 29413

Thermosynechococcus elongatus BP-1

Synechococcus Yellowstone A (JA-3-3Ab)

Synechococcus Yellowstone B (JA-2-3B'a(2-13))
Table III. Evaluation of 24 cyanobacterial genome sequences for the presence of a gene encoding an L-amino acid oxidase with similarity to the AoxA in Synechococcus elongatus PCC 6301 and for the presence of a $\operatorname{cph} A$ gene encoding a cyanophycin synthetase.

Two (+) indicate the presence of two aox-similar genes. 
for basic L-amino acids as being present in Synechococcus elongatus PCC 6301 and PCC 7942.

At the beginning of this evaluation a rather surprising and unexpected result was obtained for the cyanobacteria Synechococcus elongatus PCC 6301 and PCC 7942. The recently available complete genome sequence of these two cyanobacteria gave evidence that Synechococcus elongatus PCC 6301 and PCC 7942 contain besides the previously investigated aoxA (accession numbers YP_171306 and ZP_00164087, respectively) an additional gene with similarity to aoxA being the gene YP_171854 in Synechococcus elongatus PCC 6301 and ZP_399388 in Synechococcus elongatus PCC 7942 (Table IV). These genes have $25 \%$ identity and $37 \%$ similarity to the aox $A$ gene. The newly identified gene was named aoxB. Since the genome sequence of Synechococcus cedrorum PCC 6908 is not yet available, it can not be decided whether this cyanobacterium also possesses an aox $B$-type gene in addition to the aox $A$-type gene encoding an L-Aox catalyzing the oxidative deamination of basic L-amino acids with L-arginine as the best substrate (see Table II).

Besides Synechococcus elongatus PCC 6301 and PCC 7942, the genomes of 22 additional cyanobacteria were evaluated for the presence of an aox $A$ similar gene (Table III). The derived protein sequences were classified either as A or B depending on whether the similarity was higher to the AoxA or AoxB protein of Synechococcus elongatus PCC 6301 (Table IV). Besides the two fresh water cyanobacteria Synechococcus elongatus PCC 6301 and PCC 7942, both possessing an aoxA- and aoxB-type gene, eight further cyanobacteria contain one or two gene(s) with similarity to the aox $A$ gene. In the fresh water cyanobacterial group these are Synechocystis sp. PCC 6803 (two aoxA-similar genes - although one gene with only very minor similarity), Gloeobacter violaceus PCC 7421 (two aoxA-similar genes), Nostoc sp. PCC 7120, and Nostoc punctiforme PCC 73102. No aoxA-similar gene is present in the three investigated thermophilic cyanobacteria (Thermosynechococcus elongatus BP-1 and the two Synechococcus Yellowstone species) and also not in the mesophilic $\mathrm{N}_{2}$-fixing Anabaena variabilis ATCC 29413. In the group of the marine cyanobacteria an aoxA-similar gene is present in Synechococcus sp. CC 9605 (two aoxA-similar genes), WH 5701 and WH 7805 as well as Trichodesmium erythraeum IMS 101 (low similarity). In none of the so far sequenced Prochlorococcus species an aoxtype gene was found.

Three of the Aox-type proteins show a higher similarity to AoxA than to AoxB of Synechococcus elongatus PCC 6301 and PCC 7942 being the Aox of Nostoc sp. PCC 7120, Nostoc punctiforme PCC 73102, and one of Aox-similar proteins of Gloeobacter violaceus PCC 7421, while all the other Aox-similar proteins listed in Table IV have a higher similarity to AoxB or a distinction can not be made. The molecular masses, pI and identity/ similarity values of these Aox-type proteins are shown in Table IV, and a phylogenetic tree is given in Fig. 3.

The proteins encoded by the aox-type genes have similarity to each other (Table IV) as well as to several L-amino acid oxidases from snakes [as e.g. to the L-amino acid oxidase of Calloselasma rhodostoma (CAB71136) or Crotalus adamanteus (AAC32267)], and also to the L-amino acid oxidase of Neurospora crassa (A38314; Niedermann and Lerch, 1990). There is no significant similarity to the L-amino acid oxidase of Chlamydomonas rheinhardtii (Piedras et al., 1992; Vallon et al., 1993). A slightly higher similarity than to the snake L-amino acid oxidases exists to various monoamine oxidases (MAO), e.g. to MAO of Myxococcus xanthus DK 1622 (XP_633086), MAO of Bacillus anthracis str. Ames (YP_018569), MAO of Nocardia farcinica IFM 10152 (YP_119289), MAO of Bacillus cereus (CAA72047), MAO of human (P27338) or MAO of Mus musculus (NP_034345), and to the murine interleukin-4-inducible Fig1 protein predicted to be a flavoprotein with similaritiy to MAOs (Raibekas and Massey, 1998; Pawelek et al., 2000).

Initially, we thought that a correlation might exist between the presence of an aox $A$ gene encoding an L-Aox catalyzing the oxidative deamination of basic L-amino acids and the absence of the cyanophycin synthetase-encoding $\operatorname{cph} A$ gene (Ziegler et al., 1998) in Synechococcus species, as being the case for the fresh water cyanobacteria Synechococcus elongatus PCC 6301 and PCC 7942 as well as Synechococcus cedrorum PCC 6908 (Simon, 1987 and Table II). The presence of an aoxAtype gene in combination with the absence of a $\operatorname{cph} A$ gene was not found in any of the other cyanobacteria. This seems to be a characteristic for these three cyanobacteria - at least among the 24 cyanobacteria investigated. 


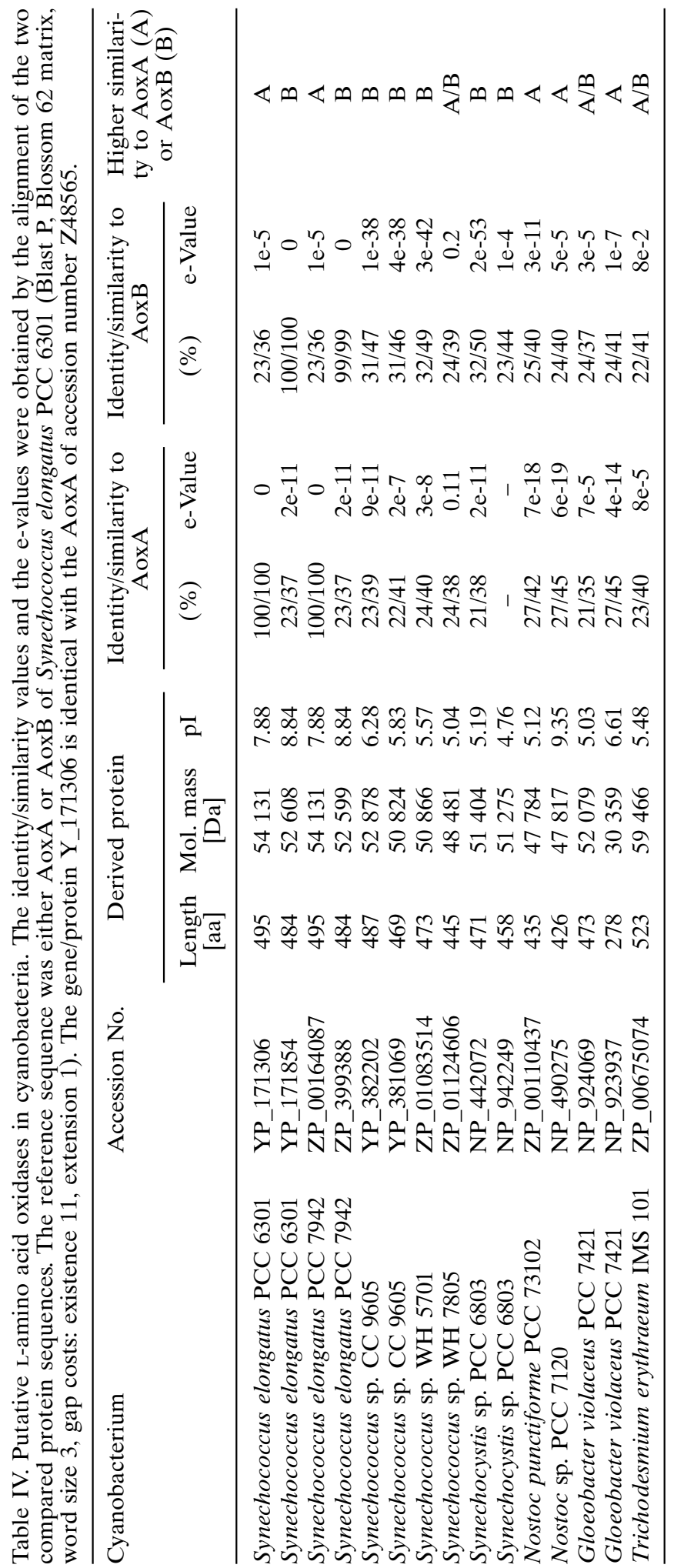

In the group of the marine cyanobacteria listed in Table III a $\operatorname{cph} A$ gene is only present in the $\mathrm{N}_{2}$-fixing Trichodesmium erythraeum IMS 101 and Crocosphaera watsonii WH 8501, but in none of the Prochlorococcus or Synechococcus species. In the group of the fresh water cyanobacteria the presence of a $\operatorname{cph} A$ gene is widespread. Only Synechococcus elongatus PCC 6301 and PCC 7942 as well as the thermophilic Synechococcus Yellowstone A (JA-3$3 \mathrm{Ab})$ do not contain a $c p h A$ gene. From the evaluation of the 24 cyanobacteria given in Table III, it can be concluded that all possible combinations with respect to an aox-type and a cphA-type gene can be found: presence or absence of both types of genes or presence or absence of one of these genes.

The evaluation given in Table III also provides evidence that in none of the analyzed Prochlorococcus species, which are minimalists with a small genome, and in none of the analyzed thermophilic cyanobacteria an aox-type gene was found. However, the majority of the remaining cyanobacteria (10 out of 15 cyanobacteria listed in Table III) contain an aox-type gene. Therefore, the function of these enzymes in the overall metabolisms of the cyanobacteria is of interest and is presently still unknown. It is well documented that the isolated L-Aox (encoded by the aoxA) of Synechococcus elongatus PCC 6301 and PCC 7942 as well as Synechococcus cedrorum PCC 6908 catalyzes the oxidative deamination of basic L-amino acids with Larginine as best substrate (Pistorius and Voss, 1980; Engels et al., 1992; Table II). Since the enzyme is in part located extracellularly, a role in utilizing extracellular Larginine as $\mathrm{N}$-source in natural environments could be assumed. However, deamination of extracellular L-arginine can not be the physiological function of this L-Aox, since the enzyme has a $K_{\mathrm{M}}$ value of 3 to $5 \mathrm{~mm}$ for $\mathrm{L}$-arginine and a very narrow substrate specificity (see Table II). L-Amino acid oxidases which are suggested to have such a function, have $K_{\mathrm{M}}$ values below $1 \mu \mathrm{M}$ and a broad specificity for L-amino acids, such as cell-surface L-amino acid oxi- 


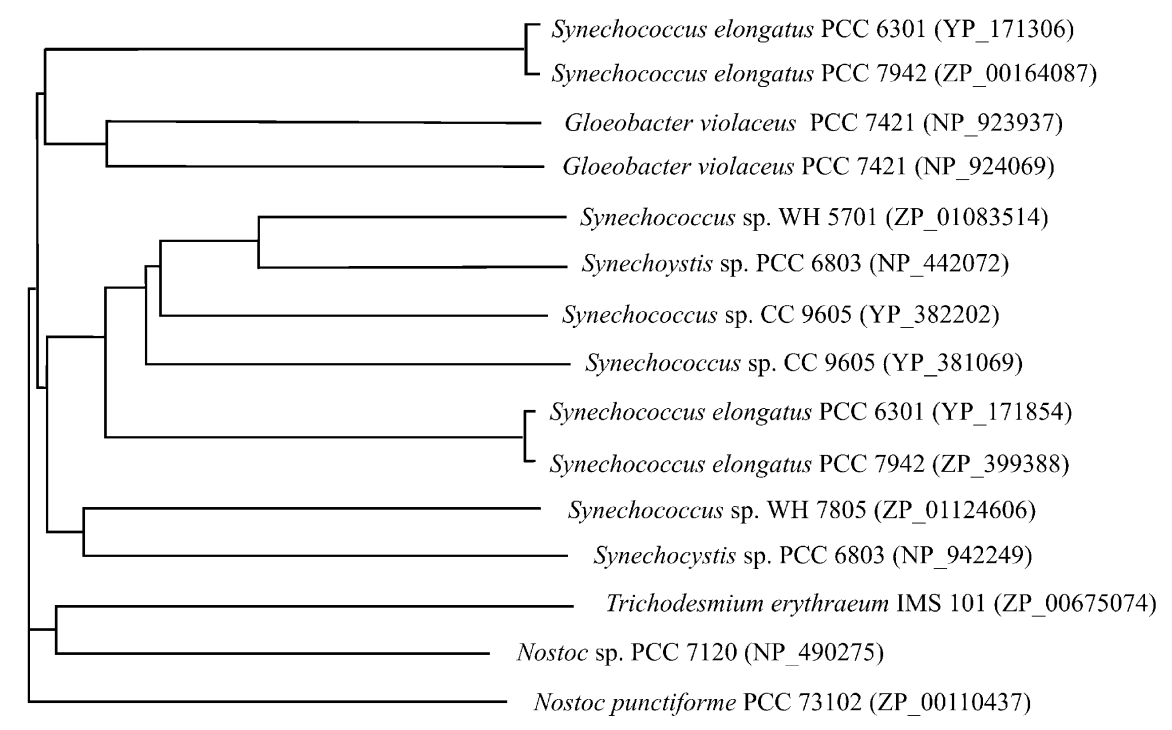

Fig. 3. Phylogenetic tree of 15 putative L-amino acid oxidases being present in the cyanobacteria shown in Tables III and IV. The phylogenetic tree was constructed on the basis of the program T-COFFE 4.45 using the Blossom 62 matrix.

dases in some marine phytoplankton genera as Pleurochrysis species (Palenik and Morel, 1990a, b). Therefore, it seems more likely that the L-Aox in Synechococcus elongatus PCC 6301 and PCC 7942 has a role in degrading L-arginine intracellularly, since excess L-arginine can not be stored in cyanophycin due to the absence of the CphA enzyme.

The function as well as the activity of all the other aox gene products (AoxA and AoxB) listed in Table IV are so far completely uncertain. On the basis of similarity evaluations the Aox-type proteins could be an L-amino acid oxidase or a monoamine oxidase. The enzymes contain a GxGxxG motif for dinucleotide binding, suggesting that AoxA as well as AoxB are FAD-binding proteins. In Synechococcus elongatus PCC 6301 and PCC 7942 both enzymes have a putative translocation pathway signal sequence with a twin-arginine motif (Berks, 1996). However, in case of AoxB this sequence deviates from the rule in two amino acids (the motif being -S-R-R-A-L-L-Ghaving $L$ instead of $F$ and $G$ instead of $K$ ) (Fig. 2). Thus, the relative distribution between intra- and extra-cellular location might be different between the AoxA and AoxB protein. In Synechocystis sp.
PCC 6803, which only contains an AoxB-type protein (and not an AoxA-type protein), we were never able to detect an L-amino acid oxidase activity by measuring the $\mathrm{O}_{2}$-uptake in the presence of L-arginine with cells grown with nitrate or with Larginine, and this holds also true for Nostoc sp. PCC 7120, which has an Aox with higher similarity to the AoxA-type protein. This would imply that these enzymes do not interact with oxygen and therefore might be L-amino acid dehydrogenases, possibly interacting with the respiratory electron chain. In $E$. coli it has been shown that an amino acid dehydrogenase can interact with the respiratory electron chain (Anraku and Gennis, 1987). The true activity of the Aox-type proteins, except for the three L-Aox proteins isolated from Synechococcus elongatus PCC 6301, Synechococcus elongatus PCC 7942, and Synechococcus cedrorum PCC 6908 (Table II), and their function are still uncertain. This aspect is presently under investigation with respect to the properties and function of the enzyme in Synechocystis sp. PCC 6803.

\section{Acknowledgement}

The financial support of the Deutsche Forschungsgemeinschaft is gratefully acknowledged. 
Abell C. W. and Kwan S.-W. (2001), Molecular characterization of monoamine oxidases A and B. Prog. Nucl. Acid Res. Mol. Biol. 65, 129-156.

Aboulmagd E., Oppermann-Sanio F. B., and Steinbüchel A. (2000), Molecular characterization of the cyanophycin synthetase from Synechocystis sp. strain PCC 6308. Arch. Microbiol. 174, 297-306.

Allen M. M. (1984), Cyanobacterial cell inclusions. Annu. Rev. Microbiol. 38, 1-25.

Altschul S. F., Gish W., Miller W., Myers E. W., and Lipman D. J. (1990), Basic local alignment search tool. J. Mol. Biol. 215, 403-410.

Anraku Y. and Gennis R. B. (1987), The aerobic respiratory chain of Escherichia coli. Trends Biochem. Sci. 12, 262-266.

Berg H., Ziegler K., Piotukh K., Baier K., Lockau W., and Volkmer-Engert R. (2000), Biosynthesis of the cyanobacterial reserve polymer multi-L-arginyl-polyL-aspartic acid (cyanophycin). Mechanism of the cyanophycin synthetase reaction studied with synthetic primers. Eur. J. Biochem. 267, 5561-5570.

Berks B. C. (1996), A common export pathway for proteins binding complex redox cofactors? Mol. Microbiol. 22, 393-404.

Bockholt R., Masepohl B., Kruft V., Wittmann-Liebold B., and Pistorius E. K. (1995), Partial amino acid sequence of an L-amino acid oxidase from the cyanobacterium Synechococcus PCC 6301, cloning and DNA sequence analysis of the aoxA gene. Biochim. Biophys. Acta 1264, 289-293.

Bockholt R., Scholten-Beck G., and Pistorius E. K. (1996), Construction and partial characterization of an L-amino acid oxidase-free Synechococcus PCC 7942 mutant and localization of the L-amino acid oxidase in the corresponding wild type. Biochim. Biophys. Acta 1307, 111-121.

Brink S., Bogsch E. G., Edwards W. R., Hynds P. J., and Robinson C. (1998), Targeting of thylakoid proteins by the $\Delta \mathrm{pH}$-driven twin-arginine translocation pathway requires a specific signal in the hydrophobic domain in conjunction with the twin-arginine motif. FEBS Lett. 434, 425-430.

Eggink G., Engel H., Vriend G., Terpstra P., and Witholt B. (1990), Rubredoxin reductase of Pseudomonas oleovorans. Structural relationship to other flavoprotein oxido-reductases based on one NAD and two FAD fingerprints. J. Mol. Biol. 212, 135-142.

Engels D. H., Engels A., and Pistorius E. K. (1992), Isolation and partial characterization of an L-amino acid oxidase and of photosystem II complexes from the cyanobacterium Synechococcus PCC 7942. Z. Naturforsch. 47c, 859-866.

Golden S. S., Nalty M. S., and Cho D.-S. C. (1989), Genetic relationship of two highly studied Synechococcus strains designated Anacystis nidulans. J. Bacteriol. 171, 24-29.

Hai T., Oppermann-Sanio F. B., and Steinbüchel A. (1999), Purification and characterization of cyanophycin and cyanophycin synthetase from the thermophilic Synechococcus sp. MA19. FEMS Microbiol. Lett. 181, 229-236.

Kachurin A. M., Golubev A. M., Geisow M. M., Veselkina O. S., Isaeva-Ivanova L. S., and Neustroev K. N.
(1995), Role of methionine in the active site of $\alpha$-galactosidase. Biochem. J. 308, 955-964.

Kouranov A. and Schnell D. J. (1996), Proteintranslocation at the envelop and thylakoid membranes of chloroplasts. J. Biol. Chem. 271, 31009-31012.

Linko P., Holm-Hansen O., Bassham J. A., and Calvin M. (1957), Formation of radioactive citrulline during photosynthetic ${ }^{14} \mathrm{CO}_{2}$-fixation by blue-green algae. J. Exp. Bot. 8, 147-156.

Michel K.-P. and Pistorius E. K. (2004), Adaptation of the photosynthetic electron transport chain in cyanobacteria to iron deficiency: The function of IdiA and IsiA. Physiol. Plant. 120, 36-50.

Montesinos M. L., Herrero A., and Flores E. (1997), Amino acid transport in taxonomically diverse cyanobacteria and identification of two genes encoding elements of a neutral amino acid permease putatively involved in recapture of leaked hydrophobic amino acids. J. Bacteriol. 179, 853-862.

Niedermann D. M. and Lerch K. (1990), Molecular cloning of the L-amino-acid oxidase gene from Neurospora crassa. J. Biol. Chem. 265, 17246-17251.

Palenik B. and Morel F. M. M. (1990a), Amino acid utilization by marine phytoplankton: A novel mechanism. Limnol. Oceanogr. 35, 260-269.

Palenik B. and Morel F. M. M. (1990b), Comparison of cell-surface L-amino acid oxidases from several marine phytoplankton. Marine Ecol. Prog. Ser. 59, 195-201.

Pawelek P. D., Cheah J., Coulombe R., Macheroux P., Ghisla S., and Vrielink A. (2000), The structure of Lamino acid oxidase reveals the substrate trajectory into an enantiomerically conserved active site. The EMBO J. 19, 4204-4215.

Piedras P., Pineda M., Munoz J., and Cárdenas J. (1992), Purification and characterization of an L-amino-acid oxidase from Chlamydomonas reinhardtii. Planta 188, 13-18.

Pistorius E. K. and Voss H. (1980), Some properties of a basic L-amino acid oxidase from Anacystis nidulans. Biochim. Biophys. Acta 611, 227-240.

Pistorius E. K., Jetschmann K., Voss H., and Vennesland B. (1979), The dark respiration of Anacystis nidulans. Production of $\mathrm{HCN}$ from histidine and oxidation of basic amino acids. Biochim. Biophys. Acta 585, 630642 .

Ponting C. P., Schultz J., Milpetz F., and Bork P. (1999), SMART: identification and annotation of domains from signalling and extracellular protein sequences. Nucleic Acids Res. 27, 229-232.

Raibekas A. A. and Massey V. (1998), Primary structure of the snake venom L-amino acid oxidase shows high homology with the mouse B cell interleukin 4-induced Figl protein. Biochem. Biophys. Res. Commun. 248, $476-478$.

Schultz J., Milpetz F., Bork P., and Ponting C. P. (1998), SMART, a simple modular architecture research tool: Identification of signaling domains. Proc. Natl. Acad. Sci. USA 95, 5857-5864.

Simon R. D. (1971), Cyanophycin granules from the blue-green alga Anabaena cylindrica: a reserve material consisting of copolymers of aspartic acid and arginine. Proc. Natl. Acad. Sci. USA 68, 265-267. 
Simon R. D. (1987), Inclusion bodies in the cyanobacteria: Cyanophycin, polyphosphate, polyhedral bodies. In: The Cyanobacteria (Fay P. and van Baalen C., eds.). Elsevier, Amsterdam, New York, Oxford, pp. 199-225.

Staden R. (1986), The current status and portability of our sequence handling software. Nucleic Acids Res. 14, 217-231.

Tabita F. R. (1994), The biochemistry and molecular regulation of carbon dioxide metabolism. In: The Molecular Biology of Cyanobacteria, Advances in Photosynthesis, Vol. 1 (Bryant D. A., ed.). Kluwer Academic Publishers, Dordrecht, Boston, London, pp. 437-467.

Tatusova T. A. and Madden T. L. (1999), Blast 2 sequences - a new tool for comparing protein and nucleotide sequences. FEMS Microbiol. Lett. 174, 247-250.

Thompson J. D., Higgins D. G., and Gibson T. J. (1994), CLUSTAL W: improving the sensitivity of progressive multiple sequence alignment through sequence weighting, position-specific gap penalties and weight matrix choice. Nucleic Acids Res. 22, 4673-4680.

Vallon O., Bulté L., Kuras R., Olive J., and Wollman F.A. (1993), Extensive accumulation of an extracellular L-amino acid oxidase during gametogenesis of Chlamydomonas reinhardtii. Eur. J. Biochem. 215, 351-360.

Wälzlein G., Gau A. E., and Pistorius E. K. (1988), Further investigations about the flavin in the L-amino acid oxidase and a possible flavin in photosystem II complexes from the cyanobacterium Anacystis nidulans. Z. Naturforsch. 43c, 545-553.

Wan J., Blakeley S. D., Dennis D. T., and Ko K. (1996), Transit peptides play a major role in the preferential import of proteins into leucoplasts and chloroplasts. J. Biol. Chem. 271, 31227-31233.

Wexler M., Bogsch E. G., Klösgen R. B., Palmer T., Robinson C., and Berks B. C. (1998), Targeting signals for a bacterial Sec-independent export system direct plant import by the $\Delta \mathrm{pH}$ pathway. FEBS Lett. 431, 339-392.

Wierenga R. K., Terpstra P., and Hol W. G. J. (1986), Prediction of the occurrence of the ADP-binding $\beta \alpha \beta$ fold in proteins, using an amino acid sequence fingerprint. J. Mol. Biol. 187, 101-107.

Yousef N., Pistorius E. K., and Michel K.-P. (2003), Comparative analysis of $i d i A$ and $i s i A$ transcription under iron starvation and oxidative stress in Synechococcus elongatus PCC 7942 wild-type and selected mutants. Arch. Microbiol. 180, 471-483.

Ziegler K., Diener A., Herpin C., Richter R., Deutzmann R., and Lockau W. (1998), Molecular characterization of cyanophycin synthetase, the enzyme catalyzing the biosynthesis of the cyanobacterial reserve material multi-L-arginyl-poly-L-aspartate (cyanophycin). Eur. J. Biochem. 254, 154-159. 